\title{
QUADRATIC SPLINE INTERPOLATION ${ }^{1}$
}

\author{
BY M. J. MARSDEN
}

Communicated by R. C. Buck, February 20, 1974

Abstract. A quadratic spline interpolation theory is developed which, in general, produces better fits to continuous functions than does the existing cubic spline interpolation theory.

1. Let $\Delta: 0=x_{0}<x_{1}<\cdots<x_{n}=1$ be a partition of $[0,1]$. A function $s$ is a spline of order $m$ having knots in $\Delta$ if $s \in C^{m-2}[0,1]$ and, on each interval $\left(x_{i-1}, x_{i}\right), s(x)$ is represented by a polynomial of degree $m-1$ or less.

For the case $m=3$, we call $s$ a quadratic spline. For quadratic splines, set $s_{i}=s\left(x_{i}\right), \lambda_{i}=s^{\prime}\left(x_{i}\right)$ for $i=0,1, \cdots, n$, and $h_{i}=x_{i}-x_{i-1}, s_{i-1 / 2}=$ $s\left(x_{i}-h_{i} / 2\right), a_{i}=h_{i+1} /\left(h_{i}+h_{i+1}\right), c_{i}=1-a_{i}$ for $i=1,2, \cdots, n$.

Any three of the parameters $s_{i-1}, s_{i-1 / 2}, s_{i}, \lambda_{i-1}, \lambda_{i}$ may be used to represent the quadratic spline $s$ on the interval $\left(x_{i-1}, x_{i}\right)$. Because of continuity, these parameters must satisfy the consistency relations

$$
a_{i} s_{i-1}+3 s_{i}+c_{i} s_{i+1}=4 a_{i} s_{i-1 / 2}+4 c_{i} s_{i+1 / 2}
$$

and

$$
c_{i} \lambda_{i-1}+3 \lambda_{i}+a_{i} \lambda_{i+1}=8\left(s_{i+1 / 2}-s_{i-1 / 2}\right) /\left(h_{i}+h_{i+1}\right)
$$

for $i=1,2, \cdots, n-1$. For simplicity, we assume that $s$ and $s^{\prime}$ are periodic, i.e.

$$
s_{0}=s_{n} \text { and } \lambda_{0}=\lambda_{n}
$$

so that (1.1) and (1.2) hold for $i=0$ and $i=n$ provided that the subscripts be read modulo $n$. For a given $\Delta$, the periodic quadratic spline subspace has dimension $n$.

2. If $y$ is a given continuous function satisfying

$$
y(0)=y(1),
$$

the periodic quadratic spline interpolant $s=S_{3} y$ associated with $y$ and $\Delta$ is

\footnotetext{
AMS (MOS) subject classifications (1970). Primary 41A10.

1 Work supported by the National Research Council of Canada while at the University of Alberta.

Copyright @ American Mathematical Society 1974
} 
uniquely defined by the conditions

$$
s_{i-1 / 2}=y_{i-1 / 2}=y\left(x_{i}-h_{i} / 2\right) \text { for } i=1,2, \ldots, n \text {. }
$$

More generally, we may define operators $S_{m}=S_{m}(\Delta, \alpha)$ by requiring that $S_{m} y$ be a periodic spline of order $m$ which interpolates $y$ at $n$ nodes specified by the parameter vector $\boldsymbol{\alpha}$.

We extend $y$ periodically with period 1 and define:

$$
\begin{gathered}
e=y-S_{3} y, \quad e_{i}=e\left(x_{i}\right), \quad\left\|f_{i}\right\|=\max \left\{\left|f_{i}\right|: 1 \leqq i \leqq n\right\}, \\
\|f\|=\max \{|f(x)|: 0 \leqq x \leqq 1\}, \\
\omega(f ; \delta)=\sup \left\{\left|f(x)-f\left(x^{\prime}\right)\right|:\left|x-x^{\prime}\right| \leqq \delta\right\}, \\
\left\|S_{3}\right\|=\sup \left\{\left\|S_{3} y\right\|:\|y\|=1\right\}, \quad \text { and } h=\left\|h_{i}\right\| .
\end{gathered}
$$

If $y \in C^{\prime}[0,1]$ with $y^{\prime}(0)=y^{\prime}(1)$, we define $e_{i}^{\prime}=e^{\prime}\left(x_{i}\right)$.

THEOREM 2.1. Let $y \in C[0,1]$ with $y(0)=y(1)$ and let $s=S_{3} y$ be the periodic quadratic spline interpolant associated with $y$ and $\Delta$. Then

$$
\begin{gathered}
\left\|s_{i}\right\| \leqq 2\|y\|, \quad\|s\| \leqq 2\|y\|, \quad\left\|S_{3}\right\| \leqq 2, \\
\left\|e_{i}\right\| \leqq 2 \omega(y ; h / 2), \quad\|e\| \leqq 3 \omega(y ; h / 2) .
\end{gathered}
$$

The bound on $\left\|S_{3}\right\|$ cannot be decreased, in general.

THEOREM 2.2 Let $y$ and $y^{\prime}$ be continuous and periodic functions. Then

$$
\begin{aligned}
& \left\|s^{\prime}\right\|=\left\|\lambda_{i}\right\| \leqq 2\left\|y^{\prime}\right\|, \quad\left\|e^{\prime}\right\| \leqq 3\left\|y^{\prime}\right\|, \quad\|e\| \leqq\left(\frac{3}{2}\right) h\left\|y^{\prime}\right\|, \\
& \left\|e_{i}\right\| \leqq h \omega\left(y^{\prime} ; h / 2\right), \quad\left\|e_{i}^{\prime}\right\| \leqq 3 \omega\left(y^{\prime} ; h / 2\right), \quad\left\|e^{\prime}\right\| \leqq\left(\frac{9}{2}\right) \omega\left(y^{\prime} ; h / 2\right), \\
& \|e\| \leqq\left(\frac{17}{8}\right) h \omega\left(y^{\prime} ; h / 2\right) \text {. }
\end{aligned}
$$

THEOREM 2.3. Let $y, y^{\prime}$, and $y^{\prime \prime}$ be continuous and periodic functions. Then

$$
\begin{aligned}
\left\|e_{i}\right\| & \leqq\left(\frac{1}{8}\right) h^{2} \omega\left(y^{\prime \prime} ; h\right) \leqq\left(\frac{1}{4}\right) h^{2}\left\|y^{\prime \prime}\right\|, \\
\left\|e_{i}^{\prime}\right\| & \leqq\left(\frac{1}{2}\right) h \omega\left(y^{\prime \prime} ; h\right) \leqq h\left\|y^{\prime \prime}\right\|, \quad\left\|e^{\prime}\right\| \leqq h \omega\left(y^{\prime \prime} ; h\right) \leqq 2 h\left\|y^{\prime \prime}\right\|, \\
\|e\| & \leqq\left(\frac{3}{8}\right) h^{2} \omega\left(y^{\prime \prime} ; h\right) \leqq\left(\frac{3}{4}\right) h^{2}\left\|y^{\prime \prime}\right\| .
\end{aligned}
$$

THEOREM 2.4. Let $y, y^{\prime}, y^{\prime \prime}$, and $y^{\prime \prime \prime}$ be continuous and periodic functions. Then

$$
\begin{aligned}
\left\|e_{i}^{\prime}\right\| \leqq\left(\frac{1}{3}\right) h^{2}\left\|y^{\prime \prime \prime}\right\|, \quad\left\|e^{\prime}\right\| \leqq\left(\frac{1}{2}\right) h^{2}\left\|y^{\prime \prime \prime}\right\|, \\
\|e\| \leqq\left(\frac{1}{4} \frac{1}{8}\right) h^{3}\left\|y^{\prime \prime \prime}\right\| \text { and }\left\|e_{i}\right\| \leqq\left(\frac{1}{8}\right) h^{3}\left\|y^{\prime \prime \prime}\right\| .
\end{aligned}
$$

If, in addition, $y^{\mathrm{iv}}$ is continuous and periodic, then

$$
\begin{aligned}
& \left\|e_{i}^{\prime}\right\| \leqq\left(\frac{1}{6}\right) h^{2}\left\|y_{i}^{\prime \prime \prime}\right\|+\left(\frac{3}{3}\right) h^{3}\left\|y^{\mathrm{iv}}\right\|, \quad\left\|e^{\prime}\right\| \leqq\left(\frac{7}{2} \frac{7}{2}\right) h^{2}\left\|y^{\prime \prime \prime}\right\|+\left(\frac{3}{3}\right) h^{3}\left\|y^{\mathrm{iv}}\right\|, \\
& \left\|e_{i}\right\| \leqq\left(\frac{1}{24}\right) h^{3}\left\|y_{i}^{\prime \prime \prime}\right\|+\left(\frac{5}{1} \frac{5}{2}\right) h^{4}\left\|y^{\mathrm{iv}}\right\|, \quad\|e\| \leqq\left(\frac{1}{9}\right) h^{3}\left\|y^{\prime \prime \prime}\right\|+\left(\frac{1}{2} \frac{1}{4}\right) h^{4}\left\|y^{\mathrm{iv}}\right\| .
\end{aligned}
$$


These theorems are established by a technique used by Meir and Sharma in their landmark cubic spline paper [6]. They hold without restriction on $\Delta$. Indeed, we may permit $x_{i-1}=x_{i}$ provided that we let $s^{\prime}$ be discontinuous at such "double knots". By setting $x_{1}=x_{0}$ and $x_{n-1}=x_{n}$, thereby producing a "triple knot", we may drop the assumptions (1.3) amd (2.1).

3. Theorem 2.1 and specific examples produce the third line in the following table of best possible norm inequalities and error bounds for low-degree $(m-1=0,1,2,3)$ spline interpolation to continuous functions on an arbitrary partition $\Delta$.

$$
\begin{array}{lll}
\text { nodes }=x_{i-1 / 2} & \left\|S_{1}\right\|=1 & \left\|y-S_{1} y\right\| \leqq \omega(y ; h / 2) \\
\text { nodes }=x_{i} & \left\|S_{2}\right\|=1 & \left\|y-S_{2} y\right\| \leqq\left(\frac{3}{2}\right) \omega(y ; h / 2) \\
\text { nodes }=x_{i-1 / 2} & \left\|S_{3}\right\| \leqq 2 & \left\|y-S_{3} y\right\| \leqq C^{*} \omega(y ; h / 2) \\
\text { nodes }=x_{i} & \left\|S_{4}\right\| \leqq \infty & \left\|y-S_{4} y\right\| \leqq \infty
\end{array}
$$

TABLE. Best bounds for arbitrary partitions

The last line of this table was established by Nord [7]. See also [3]. We conjecture that the nodes $\left(x_{i-1}+x_{i}+x_{i+1}\right) / 3$ would produce finite constants $A$ and $B$ in the relations $\left\|S_{4}\right\| \leqq A$ and $\left\|y-S_{4} y\right\| \leqq B \omega(y ; h / 2)$. These nodes have appeared previously in connection with variationdiminishing cubic spline approximation (see [5] and [4]).

4. For quadratic spline interpolation, the assertion $\|e\|=O\left(h^{3}\right)$ cannot be improved. However, on a uniform mesh with $x_{i}=i / n$ and $h_{i}=h=1 / n$, point error bounds with an extra factor of $h$ are valid. These are similar to point error bounds for cubic spline interpolation which have recently been discovered by T. R. Lucas [2]. See also [1].

Theorem 4.1. Let $\Delta=\{i / n\}$. Then $\left\|S_{3}\right\|<\sqrt{ }$. Let $\lambda=(3 \pm \sqrt{ } 3) / 6$. If $y, y^{\prime}, y^{\prime \prime}, y^{\prime \prime \prime}$, and $y^{\mathrm{iv}}$ are continuous and periodic functions, then

and

$$
\left\|e_{i}\right\| \leqq\left(\frac{5}{1} \overline{2}\right) h^{4}\left\|y^{\mathrm{iv}}\right\|, \quad\left\|e_{i-\lambda}^{\prime}\right\| \leqq\left(\frac{1}{2}\right) h^{3}\left\|y^{\mathrm{iv}}\right\|,
$$

$$
\left\|e_{i-1 / 2}^{\prime \prime}\right\| \leqq\left(\frac{11}{1}\right) h^{2}\left\|y^{\mathrm{iv}}\right\| \text {. }
$$

The assertion $\left\|S_{3}\right\|<\sqrt{ } 2$ for a uniform mesh was proved in [8]. It can also be proved by the methods of [9]. Indeed, each of the assertions of [9] has a quadratic spline counterpart.

\section{REFERENCES}

1. G. Birkhoff and C. R. de Boor, Piecewise polynomial interpolation and approximation, Approximation of Functions (Proc. Sympos. General Motors Res. Lab., 1964), Elsevier, Amsterdam, 1965, pp. 164-190. MR 32 \#6646. 
2. T. R. Lucas, Error bounds for interpolating cubic splines under various end conditions, Notices Amer. Math. Soc. 19 (1972), A-795. Abstract \#699-B15.

3. M. J. Marsden, Cubic spline interpolation of continuous functions. J. Approximation Theory 10 (1974), 103-111.

4. - - An identity for spline functions with applications to variation-diminishing spline approximation, J. Approximation Theory 3 (1970), 7-49. MR 40 \#7682.

5. M. J. Marsden and I. J. Schoenberg, On variation diminishing spline approximation methods, Mathematica (Cluj) 8 (31) (1966), 61-82. MR 35 \#4648.

6. A. Sharma and A. Meir, Degree of approximation of spline interpolation, J. Math. Mech. 15 (1966), 759-767. MR 33 \#3006.

7. S. Nord, Approximation properties of the spline fit, Nordisk Tidskr. InformationsBehandling (BIT) 7 (1967), 132-144. MR 36 \#1887.

8. F. B. Richards, Convergence of natural spline interpolations on uniform subdivisions, J. Approximation Theory (to appear).

9. F. Schurer and E. W. Cheney, On interpolating cubic splines with equally-spaced nodes, Nederl. Akad. Wetensch. Proc. Ser. A 71=Indag. Math. 30 (1968), 517-524. MR 40 \#6129.

Current address: University of Pittsburgh, Pittsburgh, Pennsylvania 15260 